\title{
Physiotherapy in the Treatment of Infantile Paralysis
}

\author{
Extracts from a paper read by Miss E. Eeg, a member of the staff of the National Foundation for \\ Infantile Paralysis, Denmark, to students of the School of Physiotherapy, University of Witwatersrand, \\ Johannesburg, in October, 1948.
}

\section{INTRODUCTION}

Before I start to tell you how the post-treatment of poliomyelitis patients is carried out in my country - Denmark - I would like to explain briefly how we happen to be in the position we are to-day. among the few countries which have a National Foundation for Infantile Paralysis. I would also like to tell you about the Institute for Research and Therapy, which belongs to the Foundation. By so doing, my explanaiion of the treatments which are given there will have a better background.

Some years ago, Danish sufferers with poliomyelitis realised that, in America, hope and encouragement was offered to their fellow sufferers. In that country, physical training was carried out for many years in the belief that affected muscles could often recover if not completely - then to an appreciable extent. The Danes themselves formed a small association which gradually expanded until in May, I945, it National Foundation for Infantile Paralysis was formed under the patronage of Queen Ingrid. The National Committee included many of the leading doctors in Orthopaedics, Physical Medicine and Research. The Government accorded the movement its support and tremendous enthusiasm was shown by the public. As a result of the generous financial support received, the Foundation was enabled to send Dr. Clemmeson, who is in charge of the Massage Dept. of the Municipal Hospital, Copenhagen and two Orthopaedic surgeons to America to undergo a stucly course. These were later followed by the physiotherapist in charge.

The Foundation established its headquarters in a large house in Copenlagen which was opened in October, I945, as the Institute for Research and Therapy. Originally, four girls, including myself, were transferred from the Municipal Hospital. On his return from America, Dr. Clemmeson was placed in charge of the Institute and at once started a course of instruction. To-day, the Massage Dept. has a staff of three doctors and more than twenty physiotherapists.

In the bcginning, treatment was based on American methods, information digested from the newest books on poliomyelitis and from our previous knowledge of the disease. As things developed, our methods gradually improved, and we received inspiration and new ideas from visiting doctors and physiotherapists from other countries. Eventually, the clinic developed more of an international than a Danish nature.. Early in 1946 patients were received from Norway. Sweden and Finland. Later they arrived from countries even further afield.
Foundation doctors and physiotherapists who lectured to Danisl and foreign students were invited to lecture in France, Holland, Sweden and Finland. In 1948 three English physiotherapists visited the clinic for three montlis in exchange for three Foundation physiotherapists. Sister Kenny lectured to us and presented an instructional film on her methods of treatment.

\section{TREATMENT}

I think it is now time to tell you about the details of our work. There are many different opinions on how to treat poliomyelitis. From our accumulated knowledge we realised that the treatment was basically the same in most countries although the details varied. Our treatment was therefore based on exercises, massage, electrotherapy and hydrotherapy. Whether we combined these treatments or concentrated on one or two types depended on the nature and length of time for which the patient had been affected. Some patients receive treatment soon after the onset whilst others attend for the first time ten to twenty years after they have contracted the disease. That we treat such long stancling cases may seem surprising but we believe that muscle strength can in many cases be regained and developed many years after the onset of the disease. Even if this is not accomplished, it is felt that something can always be done to make life easier for the chronic case. And now to the actual treatment, so we will start with exercises.

Exercises.-Firstly, we make it a general rule not to waste time by giving exercises which are either too easy or too difficult. Exercises are given to improve co-ordination and to help patients overcome difficulties in their work or daily life. The main group is given to develop strength in the affected muscles, so we will talk about them first.

Exercises for Muscle Strength.-When all the muscles in a certain group are affected to the same degree, it is a fairly simple matter to find specific exercises directed to improving muscle strength. As an example, let us consider a case in which the left hip extensors are affected.

If these muscles can complete a full range of movement against resistance, the patient should be placed in the prone position with the legs straight. Full active extension of the hip should be performed against resistance applied above and behind the knee. The pelvis should be stabilised to ensure that he does not hollow his back. If the muscles are too weak to perform this action. against resistance, the same exercise is given as a free movement. If the muscles 
are so weak that they cannot overcome gravity, are patient should be placed on his normal side. Hip extension can now take place with gravity eliminated.

Let us now deal with the case whose hip extensors are affected in different degrees. For example, let us are ame that gluteus maximus is affected whilst the lamstring muscles are normal. In this case, hip extension is given with the knee flexed thus decreasing the action of the hamstrings. The condition of the the acle would determine whether resistance should be used or gravity eliminated. This exercise is called an isolated gluteus maximus exercise and brings us to one of our greatest problems, i.e., that of devising and giving isolated muscle exercises.

Isolated Muscle Exercises.-The chief purpose served by these exercises is to decrease the action of normal synergists in an effort to get the affected muscle to work. How to accomplish this when the synergists are stronger - or not affected at all has been explained in the previous example. In that case, gluteus maximus was isolated by flexing the knee. Had the exercise been given with the knee extended, the normal hamstring muscles would have performed all, or at least most of the muscle work involved in extending the hip and gluteus maximus would have been given little if any exercise. It will be seen therefore that isolated muscle exercises are of the greatest importance and if possible should always be included in the scheme of treatment.

[Miss Eeg then demonstrated the methods used to isolate various muscles and went on to state that the isolation of muscles probably required more specialised knowledge and skill on the part of the operator than any other single factor in the physiotherapeutic treatment of the disease.]

Conducted Exercises. - I will now deal with another group of exercises - the Conducted exercises. These are used only to develop very weak muscles or those in which a contraction can neither be seen nor felt. As an example let us take a case where there is no contractability in the hamstrings. The main point is to teach the patient to feel these muscles as they are passively moved right from the start of treatment. To assist him to do this, he should be shown a diagram of the knee flexors, and the mechanism through which they flex the knee under normal conditions should be explained to him for the first few seances. The patient should then be placed in the prone position whilst the operator bends the knee up and clown in a slow and rhythmical manner, the speed being dictated by the patient. He is instructed to concentrate on the bending movement, to picture in his mind the mechanism and to try and actually "feel" the movement. For the first few seances, conducted exercises can only be given for a very short time as the patient tires very easily. Later, they can be carried on for increasingly longer periods - say three to five minutes - after which massage is given and the exercises may be continued. In many cases, this type of exercise is used in combination with electrical stimulation. Three stimuli are given whilst the operator counts I-2-3; the patient thên attempts the movement whilst the operator continues to bend the knee to the same rhythm of $4-5-6$.

Exercises for Co-ordination.-A great variety of this type of exercise is given to help the patients in their daily life, e.g., to ascend -steps, to use crutches or sticks and to get up from a wheel-chair, etc. For this latter action a whole series of exercises is available, the chief purpose of which is to strengthen the shoulder and arm muscles particularly triceps. In addition to these, all patients, whatever their degree of disablement, receive a general training for their normal muscles.

A large number of corrective walking exercises are taught, especially to those long standing cases who have 110 w recovered, but through force of habit continue to walk in an ungainly fashion.

\section{ELECTRICAL TREATMENT}

Electrical treatment is used extensively in Denmark in the treatment of poliomyelitis. The apparatus used is known as a Myotensor and was designed by Dr. Clemmeson in collaboration with two Danish engineers. This apparatus has certain technical advantages over both galvanic and faradic apparatuses.

[Miss Eeg then gave a few comparative details in support of this contention.]

In many cases which had previously shown no response to galvanic stimulation, a strong and painless contraction was produced with the Myotensor. This is of great value in the treatment of children. In cases with only slight paresis, a large number of the motor units possess an unimpaired innervation and require no electrical stimulation except possibly for the circulatory effect produced.

In severe cases, electrical stimulation offers the advantage that it permits the isolated training of affected muscles during the period when their active innervation is still sluggish. When such stimulation is given regularly, the patient will - to some extent retain the sensation of contraction and it is easier for him to "find the innervation" when muscle power returns. A certain amount of training of partially paretic or weak muscles is effected in children and in patients who are not sufficiently energetic or lack co-ordination

\section{HYDROTHERAPYY}

In using hydrotherapy, the water should be slightly below body temperature thus avoiding exhaustive symptoms. Hydrotherapy is used for two purposes:-

( I) To develop muscle strength by exercising - in the Hubbard tank.

(2) To increase the circulation before treatment with the aid of hot showers and local baths.

Care is taken not to waste time or occupy the baths unnecessarily by exercising muscles which are strong enough to work without the support of water. Seriously affected muscles are invariably tested for voluntary response in the tank as they often reveal a response in water which has not previously been apparent. Massage is given only to the weakest muscles for its stimulating effect and to improve the circulation. Only a gentle petrissage is used, care being taken not to stretch or injure the weak tissues.

\section{MANIPULATION}

This form of treatment was included in our scheme in I947. Beneficial results were obtained in the manipulation of ankle and foot joints. These were the only joints in which manipulation was attempted.

\section{SPASM AND CONTRACTURES}

In Denmark, spasm is 'seldom as apparent as it is in this country or in America. When a patient is reported as exhibiting this symptom, the hospital concerned, wherever in Denmark it may be, calls for a 
physiotherapist from the Foundation. She has been trained in the Kenny method of applying hot packs to patients in the acute stage, and her job is to instruct the nurses in this method. As we have so little experience with this type of case, I am unable to give you any further information on this subject.

With contractures, lowever, the position is quite different. With the exception of the more recent cases who have had the advantage of correct nursing and treatment, nearly all the cases we have received have had at least one contracture. These cases are given repeated contracture stretchings during the course of their treatment, regardless of the strength of the contracted muscles, because we have learnt from experience that a greater degree of muscle strength can be attained in weak contracted muscles which have been stretched than wonld otherwise be the casc.

I would like to finish this talk by expressing the hope that a foundation will be formed in South Africa, not for research only, but for treatment. In Denmark, all cases receive free the specialised treatment which their condition merits. New cases are taken in hand immediately and contact is maintained with chronic cases. Local doctors and physiotherapists assist by sending these latter twice yearly to the Institute for consultation. In case of indigence, the benefit society - to which it is compulsory for all Danes to belong - co-operates with the Foundation to pay expenses.

The Foundation has established three specially trained doctors in widely separate centres. These act as advisers in all matters pertaining to poliomyelitis. At regular intervals, courses of instruction are held for doctors, nurses and physiotherapists.

One final point which I would like to make concerns our Institute for Research and Therapy. As we deal witl one condition only, a vast amount of research is done on the pathology and treatment of poliomyelitis. The therapy staff acquires a confidence in their knowledge and treatment of this condition which is reflected in the excellent results which are obtained.

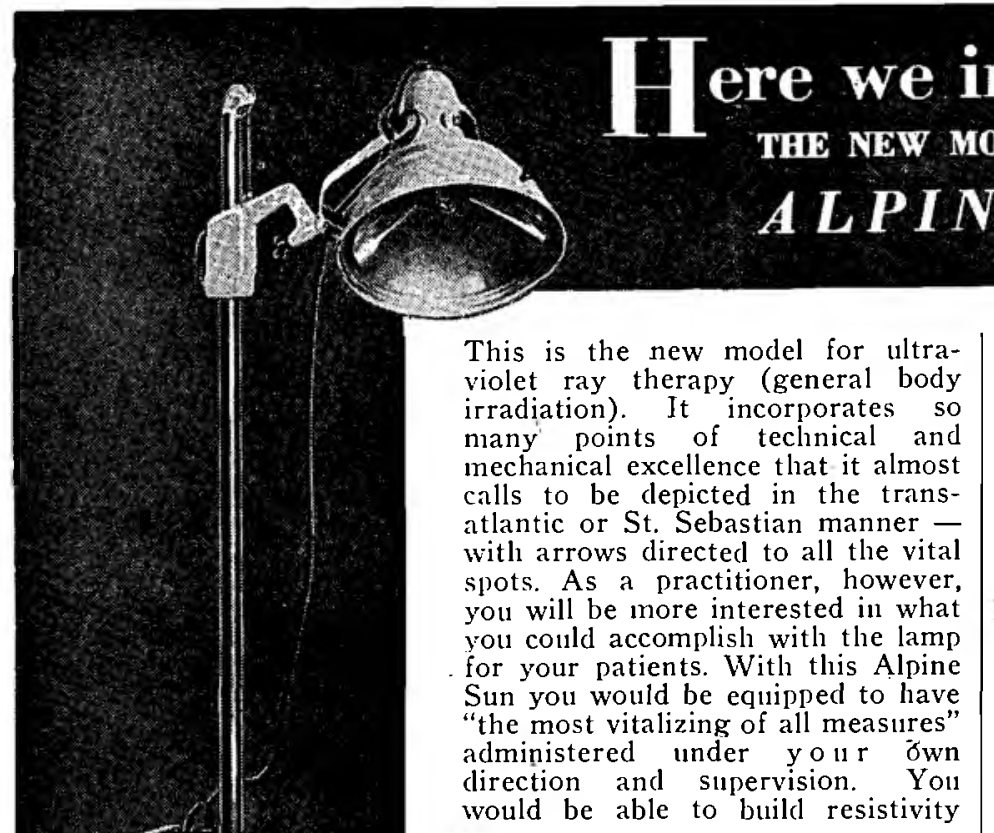

and develop reactive capacity, assisting other forms of treatment. And you would be applying this protean therapy in the most effective and economical manner possible for whilst the cost of this unit is moderate, no better lamp can bc bought at any price. Just write for our leaflet Mi59 (or post this colipon)

\section{IIANO VIA LTH. SLOUGH, ENGLAND \\ The Specialists in Actinotherapy Equlpment \\ Represented throughoul. Southern Africa by}

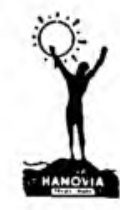

THE BRITISH GENERAL ELECTRIC CO., LTD. “MAGNET HOUSE" C/o Loveday \& Anderson Sts., JOHANNESBURG Branches: CAPE TOWN, DURBAN, PORT ELIZABETH, SALISBURY, BULAWAYO To: The British General Electric Co. Ltd., P.O. Box 2406, Johannesburg Please send me Leaflet describing No. 159 "THE ALPINE SUN LAMP" Name.

Address. 